\title{
AN UPPER BOUND FOR THE SUM OF LARGE DIFFERENCES BETWEEN PRIME NUMBERS
}

\author{
R. J. COOK
}

\begin{abstract}
Let $p_{n}$ denote the $n$th prime number, $d_{n}=p_{n+1}-p_{n}$. We estimate the sum $\sum d_{n}$ taken over $p_{n}<x, d_{n}>x^{\mu}$ where $1 / 6<\mu<5 / 9$.
\end{abstract}

1. Introduction. There has been much work done on studying irregularities in the distribution of prime numbers. Let $p_{n}$ denote the $n$th prime number and set $d_{n}=p_{n+1}-p_{n}$. Assuming the Riemann hypothesis Cramér [3] proved that $d_{n}=$ $O\left(p_{n}^{1 / 2} \log p_{n}\right)$ and [4] that for any $\varepsilon>0$

$$
\sum_{p_{n}<x} d_{n}^{2} \ll x \log ^{3+\varepsilon} x
$$

where the constant implied by Vinogradov's notation depends only on $\varepsilon$, and Selberg [17] subsequently improved on this.

For $0 \leqslant \mu \leqslant 1$ let

$$
S_{\mu}(x)=\sum_{p_{n}<x, d_{n}>x^{\mu}} d_{n}
$$

and take $f(\mu)$ to be the least value such that for all $\varepsilon>0$,

$$
S_{\mu}(x) \ll x^{f(\mu)+\varepsilon} \quad \text { as } x \rightarrow \infty .
$$

From (1) we see that the Riemann hypothesis implies

$$
f(\mu) \begin{cases}=0 & \text { for } \mu>1 / 2, \\ \leqslant 1-\mu & \text { for } 0 \leqslant \mu<1 / 2 .\end{cases}
$$

Montgomery [15, pp. 130-132] has shown that the density hypothesis is sufficient to imply that $f(\mu)=0$ for $\mu>1 / 2$.

Since the Riemann hypothesis implies that $d_{n}=O\left(p_{n}^{1 / 2} \log p_{n}\right)$, Erdös asked whether $f(1 / 2)<1$ and there has been much interest recently in estimating $f(1 / 2)$ (see [2], [11], [12], [16] and [20]). Heath-Brown [7] has given an unconditional proof that

$$
\sum_{p_{n}<x} d_{n}^{2} \ll x^{4 / 3}(\log x)^{10,000}
$$

which gives

$$
f(\mu) \begin{cases}=0 & \text { for } \mu>2 / 3 \\ \leqslant 4 / 3-\mu & \text { for } 2 / 3>\mu>1 / 3\end{cases}
$$

Received by the editors March 13, 1978 and, in revised form, November 9, 1979.

AMS (MOS) subject classifications (1970). Primary $10 \mathrm{H} 15$.

Key words and phrases. Prime number, density theorem. 
while Huxley [10] has shown that $f(\mu)=0$ for $\mu>7 / 12$.

A recent result of Warlimont [19], when combined with Huxley's density theorem [10], shows that $f(\mu)<1$ for $\mu>1 / 6$. Warlimont's result depends on an inexplicit estimate of Halász and Turán [6] and so seems incapable of giving explicit upper bounds for $f(\mu)$ in the range $\mu>1 / 6$. We use different estimates to improve the upper bound (3) throughout the interval $5 / 9>\mu>1 / 6$.

THEOREM. We have $f(\mu) \leqslant F(\mu)$ where

$$
F(\mu)= \begin{cases}19 / 18-\mu / 2 & \text { for } 47 / 99<\mu<5 / 9 \\ 10 / 7-9 \mu / 7 & \text { for } 3 / 8<\mu<47 / 99 \\ 1-\mu / 7 & \text { for } 7 / 32<\mu<3 / 8 \\ 11 / 10-3 \mu / 5 & \text { for } 1 / 6<\mu<7 / 32\end{cases}
$$

In particular, $f(1 / 2) \leqslant 29 / 36=.805$ which is an improvement on Ivić's estimate [13], $f(1 / 2) \leqslant .809$.

I am grateful to Roger Heath-Brown, Martin Huxley and Aleksander Ivić for making manuscripts available prior to publication; I am indebted to the referee for many helpful suggestions and for pointing out a gap in my original argument.

2. Preliminaries. Let $\varepsilon>0$ and $x>x_{0}(\varepsilon)$ be large. It is sufficient to obtain the corresponding estimates for a sum

$$
\sum_{\substack{x<p_{n}<2 x \\ d_{n}>x^{\mu}}} d_{n}
$$

since the theorem then follows on summing over intervals $[x, x / 2],[x / 2, x / 4], \ldots$ and noticing that at $O(\log x)$ end-points we have $d_{n}=O\left(x^{7 / 12+e}\right)$ : see Huxley [10].

The function

$$
\psi(z)=\sum_{p^{r}<z} \log p
$$

has an explicit formula (see Chandrasekharan [1, p. 120])

$$
\psi(z)=z-\sum_{|\gamma|<T} z^{\rho} / \rho+E(z, T)
$$

where the summation is taken over the nontrivial zeros $\rho=\beta+i \gamma$ of $\zeta(s)$, $0<\alpha \leqslant 1$ and $E(z, T)=O\left(z T^{-1} \log ^{2} z\right)$ uniformly over the range $3<T<z$.

We take

$$
T=x^{\alpha}, \quad U=2 x^{1-\mu}, \quad \delta=\log (1+1 / U) \ll U^{-1},
$$

and put

$$
\Delta(y)=\psi\left(y+\frac{y}{U}\right)-\psi(y)-\frac{y}{U}+\sum_{(8)} \frac{\left(e^{\delta \rho}-1\right)}{\rho} y^{\rho}-E\left(y+\frac{y}{U}\right)+E(y)
$$

where $x<y \leqslant 2 x$, the summation is over the zeros of $\zeta(s)$ in the region

$$
|\gamma| \leqslant x^{\alpha}, \quad \sigma_{0}<\beta<1
$$


and $\sigma_{0}=\sigma_{0}(\mu)$ will be chosen later. From (5) we have

$$
\Delta(y)=\sum_{(10)} \frac{\left(e^{\delta \rho}-1\right)}{\rho} y^{\rho}
$$

where the summation is over the zeros of $\zeta(s)$ in the region

$$
|\gamma| \leqslant x^{\alpha}, \quad 0<\beta<\sigma_{0} .
$$

Following Warlimont [19] we have

$$
\int_{x}^{2 x}|\Delta(y)|^{2} d y \ll \delta^{2} x \log ^{2} x \sum_{(10)} x^{2 \beta} \ll U^{-2} x \log ^{2} x \sum_{(10)} x^{2 \beta} .
$$

The proof of the theorem depends on obtaining a lower bound for $\int|\Delta(y)|^{2} d y$ in terms of large differences between primes, and an upper bound for the right side of (11) via zero-density estimates.

Let $N(\sigma, T)$ denote the number of zeros of $\zeta(s)$ in the rectangle $\sigma<\beta<1$, $|\gamma| \leqslant T$ and write $N(T)$ for $N(0, T)$. Then, see Davenport [5, Chapter 15], we have

$$
N(T) \ll T \log T .
$$

Since

$$
\begin{aligned}
\sum_{(10)}\left(x^{2 \beta}-1\right) & =2 \sum_{(10)} \int_{0}^{\beta} x^{2 \sigma} \log x d \sigma \\
& =2 \int_{0}^{\sigma_{0}} x^{2 \sigma} N\left(\sigma, x^{\alpha}\right) \log x d \sigma
\end{aligned}
$$

we have

$$
\sum_{(10)} x^{2 \beta} \ll x^{1+\alpha} \log x+\int_{1 / 2}^{\sigma_{0}} x^{2 \sigma} N\left(\sigma, x^{\alpha}\right) \log x d \sigma .
$$

3. Density theorems. These are upper bounds for $N(\sigma, T)$ which can be stated in either the form

$$
N(\sigma, T) \ll T^{g(\sigma)(1-\sigma)} \log ^{A} T
$$

where $A$ is an absolute constant, not necessarily the same at each occurrence, or in the form

$$
N(\sigma, T) \ll T^{g(\sigma)(1-\sigma)+\eta}
$$

where $\eta$ is an arbitrary small positive constant. In (14) and (15) the implicit constants are independent of $\sigma$, although they may depend on $A$ and $\eta$.

LEMMA 1. For $1 / 2<\sigma<1$, (14) holds with $g(\sigma)=3 /(2-\sigma)$.

This is due to Ingham [12].

LEMMA 2. For $3 / 4 \leqslant \sigma \leqslant 1$, (14) holds with $g(\sigma)=3 /(3 \sigma-1)$.

This is due to Huxley [10].

LEMMA 3. For $3 / 4 \leqslant \sigma \leqslant 11 / 14$, (15) holds with $g(\sigma)=1 /(7 \sigma-5)$.

This is the case $k=3$ of equation (1.8) of Jutila [13]. 
LEMMA 4. For $11 / 14 \leqslant \sigma \leqslant 1$, (15) holds with $g(\sigma)=9 /(7 \sigma-1)$.

This is due to Heath-Brown [9].

LEMma 5. Let $M(\psi, T)=\max |\zeta(s)|$ where the maximum is taken over re $s>\psi$, $|\mathrm{im} s|<T$ and $|s-1|>1$. Then for $T>2$

$$
N(\sigma, T) \ll\left(B M(\psi, 8 T) \log ^{5} T\right)^{2(1-\sigma)(3 \sigma-1-2 \psi) /(2 \sigma-1-\psi)(\sigma-\psi)} \log ^{8} T
$$

provided that $1 / 2 \leqslant \psi \leqslant 1$ and $\sigma>(1+\psi) / 2$, where $B$ is a large absolute constant.

This is Theorem 12.3 of Montgomery [15].

We combine Lemma 5 with estimates for $\zeta(s)$ obtained by van der Corput's method.

LEMMA 6. If $l \geqslant 3, L=2^{l-1}, \sigma=1-l /(2 L-2)$ then

$$
\zeta(\sigma+i t) \ll t^{1 /(2 L-2)} \log t .
$$

This is Theorem 5.14 of Titchmarsh [18].

LEMMA 7. For $17 / 18 \leqslant \sigma \leqslant 1$, (14) holds with $g(\sigma)=1 /(7 \sigma-6)$.

Proof. Taking $l=4$ in Lemma 6 we have

$$
\zeta(5 / 7+i t) \ll t^{1 / 14} \log t .
$$

This implies that the function

$$
g(s)=\frac{\zeta(s)}{s^{1 / 14} \log s}\left(\frac{s-1}{s}\right)^{2}
$$

which is holomorphic for re $s>1$, is uniformly bounded on the line re $s=5 / 7$. It is also uniformly bounded in the half-plane re $s>2$, so by the Phragmen-Lindelöf theorem it is uniformly bounded in re $s \geq 5 / 7$. Hence

$$
M(5 / 7,8 T) \ll T^{1 / 14} \log T \text {. }
$$

Now apply Lemma 5 with $\psi=5 / 7$. For $17 / 18<\sigma<1$ the exponent of $B$ is bounded uniformly in $\sigma$ so

$$
g(\sigma) \leqslant \frac{2(3 \sigma-1-10 / 7)}{14(2 \sigma-1-5 / 7)(\sigma-5 / 7)}=\frac{(21 \sigma-17)}{2(7 \sigma-6)(7 \sigma-5)}<\frac{1}{(7 \sigma-6)} .
$$

Combining these lemmas, we have

$$
N(\sigma, T) \ll T^{G(\sigma)(1-\sigma)+\eta} \quad(1 / 2<\sigma<1)
$$

where

$$
G(\sigma)= \begin{cases}3 /(2-\sigma), & 1 / 2 \leq \sigma<3 / 4, \\ 3 /(3 \sigma-1), & 3 / 4 \leqslant \sigma<7 / 9 \\ 1 /(7 \sigma-5), & 7 / 9 \leqslant \sigma<11 / 14 \\ 9 /(7 \sigma-1), & 11 / 14<\sigma<53 / 56 \\ 1 /(7 \sigma-6), & 53 / 56<\sigma<1 .\end{cases}
$$


Thus $G(\sigma)$ is a continuous function with $G(1 / 2)=2$, increasing monotonically to the value $G(3 / 4)=12 / 5$ and thereafter decreasing monotonically to the value $G(1)=1$.

4. Estimation of an integral. For given $\mu$ satisfying $1 / 6<\mu<5 / 9$ we choose $\sigma^{*}=\sigma^{*}(\mu)$ to be the largest $\sigma^{*}<1$ satisfying $(1-\mu) G\left(\sigma^{*}\right)=1$. Thus

$$
\sigma^{*}=\sigma^{*}(\mu)=\left\{\begin{array}{lc}
(7-\mu) / 7, & 1 / 6<\mu<3 / 8, \\
(10-9 \mu) / 7, & 3 / 8<\mu<1 / 2, \\
(6-\mu) / 7, & 1 / 2<\mu<5 / 9
\end{array}\right.
$$

We take $\alpha=1-\mu+\varepsilon_{1}$ where $\varepsilon_{1}>0$ is a constant which will be chosen later. For a given small $\varepsilon_{2}>0$ we take $\sigma_{0}=\sigma_{0}(\mu)$ to be the largest $\sigma_{0}<1$ satisfying

$$
\alpha G\left(\sigma_{0}\right)=1-\varepsilon_{2} \text {. }
$$

Since $G(\sigma)$ is a continuous decreasing function near $\sigma=1$, for given $\delta>0$ we have $0<\sigma_{0}-\sigma^{*}<\delta$ provided that $\varepsilon_{1}$ is chosen sufficiently small in terms of $\delta$, and $\varepsilon_{2}$ sufficiently small in terms of $\varepsilon_{1}$.

LemMa 8. For any $\varepsilon>0$ we can choose $\delta, \varepsilon_{1}, \varepsilon_{2}$ so that

$$
\int_{1 / 2}^{\sigma_{0}} x^{2 \sigma} N\left(\sigma, x^{\alpha}\right) \log x d \sigma \ll x^{1+F(\mu)+\varepsilon} \text { as } x \rightarrow \infty
$$

Proof. We break the range of integration up into subintervals $[1 / 2,3 / 4]$, $[3 / 4,7 / 9],[7 / 9,11 / 14],[11 / 14,53 / 56]$ and $[53 / 56,1]$, stopping at $\sigma_{0}$. We use the upper bounds for $N\left(\sigma, x^{\alpha}\right)$ provided by (18), and note that the exponent is a continuous function of $\alpha$ and $\sigma$, and so uniformly continuous on the region $1 / 2<\sigma \leqslant 1,0 \leqslant \alpha \leqslant 1$.

Putting $a=1-\mu$ it is sufficient to prove that for any $\varepsilon>0$

$$
\int_{1 / 2}^{\sigma^{*}} x^{2 \sigma} N\left(\sigma, x^{a}\right) \log x d \sigma \ll x^{1+F(\mu)+e / 2} \text { as } x \rightarrow \infty,
$$

as the perturbations from $\sigma^{*}$ to $\sigma_{0}$ and $a$ to $\alpha$ can be absorbed in the exponent $\varepsilon$ provided that $\varepsilon_{1}, \varepsilon_{2}$ and $\delta$ are sufficiently small as functions of $\varepsilon$. For we estimate the integrand in (21) as

$$
\ll x^{2 \sigma} x^{a G(\sigma)(1-\sigma)+\eta} \log x .
$$

Between $\sigma^{*}$ and $\sigma_{0}$ the function $G(\sigma)(1-\sigma)$ decreases so this is

$$
\ll x^{2 \sigma_{0}+a G\left(\sigma^{*}\right)\left(1-\sigma^{*}\right)+\eta} \log x \ll x^{2 \sigma_{0}+\left(1-\sigma^{*}\right)+\eta} \log x \ll x^{1+\sigma^{*}+2 \delta+\eta} \log x
$$

and $\sigma^{*}(\mu) \leqslant F(\mu)$ for $1 / 6 \leqslant \mu \leqslant 5 / 9$. Replacing $a=1-\mu$ by $\alpha=1-\mu+\varepsilon_{1}$ in the estimates for the integrand will increase the exponents of $x$ by a factor no larger than $1+3 \varepsilon_{1}$, since $1-\mu \geqslant 1 / 3$. Thus the lemma follows from the estimate (21).

The first interval contributes

$$
\ll \max _{1 / 2<\sigma<3 / 4} x^{2 \sigma+3 a(1-\sigma) /(2-\sigma)+\eta} \ll x^{3 / 2+3 a / 5+\eta},
$$

since the exponent is an increasing function of $\sigma$. 
The second interval contributes

$$
\ll \max _{3 / 4<\sigma<7 / 9} x^{2 \sigma+3 a(1-\sigma) /(3 \sigma-1)+\eta} \text {. }
$$

The exponent is a convex function of $\sigma$ so the maximum occurs at an end-point of the interval. Hence the contribution of the second interval is

$$
\begin{aligned}
& \ll \max \left(x^{3 / 2+3 a / 5+\eta}, x^{14 / 9+a / 2+\eta}\right) \\
& \ll \begin{cases}x^{3 / 2+3 a / 5+\eta}, & a>5 / 9, \\
x^{14 / 9+a / 2+\eta}, & a<5 / 9 .\end{cases}
\end{aligned}
$$

The third interval contributes

$$
\ll \max _{7 / 9<\sigma<11 / 14} x^{2 \sigma+a(1-\sigma) /(70-5)+\eta}
$$

and again the exponent is a convex function of $\sigma$ so this is

$$
\ll \max \left(x^{14 / 9+a / 2+\eta}, x^{11 / 7+3 a / 7+\eta}\right) \ll x^{14 / 9+a / 2+\eta}
$$

since $a=1-\mu>2 / 9$.

For $4 / 9<a<1 / 2, \sigma^{*} \in[7 / 9,11 / 14]$ and the total contribution of the interval $\left[1 / 2, \sigma^{*}\right]$ is $\ll x^{14 / 9+a / 2+\eta}$.

For $1 / 2 \leqslant a \leqslant 5 / 8, \sigma^{*} \in[11 / 14,53 / 56]$ and the contribution of the interval $\left[11 / 14, \sigma^{*}\right]$ is

$$
\begin{aligned}
& \ll \max \left(x^{11 / 7+3 a / 7+\eta}, x^{2 \sigma^{*}+9 a\left(1-\sigma^{*}\right) /\left(7 \sigma^{*}-1\right)+\eta}\right) \\
& =\max \left(x^{11 / 7+3 a / 7+\eta}, x^{1+\sigma^{*}+\eta}\right) .
\end{aligned}
$$

Now $\sigma^{*}=(1+9 a) / 7$ so the total contribution of the interval $\left[1 / 2, \sigma^{*}\right]$ is

$$
\ll \begin{cases}x^{14 / 9+a / 2+\eta}, & 1 / 2<a<52 / 99, \\ x^{1+\sigma^{*}+\eta}, & 52 / 99<a<5 / 8 .\end{cases}
$$

For $a>5 / 8$ the contribution of the interval $[1 / 2,53 / 56]$ is

$$
\ll \begin{cases}x^{53 / 28+3 a / 35+\eta}, & a<55 / 72, \\ x^{3 / 2+3 a / 5+\eta}, & a>55 / 72 .\end{cases}
$$

Now $\sigma^{*}=(6+a) / 7$ and the contribution of the interval $\left[53 / 56, \sigma^{*}\right]$ to the integral is

$$
\ll \max \left(x^{53 / 28+3 a / 35+\eta}, x^{1+\sigma^{*}+\eta}\right) \ll x^{1+\sigma^{*}+\eta}=x^{(13+a) / 7+\eta}
$$

since $a>5 / 8$. Thus for $a \geqslant 5 / 8$ the integral [1/2, $\left.\sigma^{*}\right]$ contributes

$$
\ll \begin{cases}x^{3 / 2+3 a / 5+\eta}, & a>25 / 32, \\ x^{(13+a) / 7+\eta}, & a<25 / 32,\end{cases}
$$

and this completes the proof of the lemma.

From Lemma 8, (11) and (13) we have

$$
\int_{x}^{2 x}|\Delta(y)|^{2} d y \ll x^{2 \mu-1+e}\left\{x^{1+\alpha}+x^{1+F(\mu)}\right\} \ll x^{2 \mu+F(\mu)+e}
$$

provided that $\varepsilon_{1}$ is sufficiently small that $\alpha<F(\mu)$, as $F(\mu)>1-\mu$ for $1 / 6<\mu$ $<5 / 9$. 
5. Estimation of a sum. Suppose that $x<p_{m}<2 x$ and $p_{m+1}-p_{m}>x^{\mu}$. Then for

$$
x<p_{m}<y<p_{m}+d_{m} / 3<p_{m+1}<2 x
$$

we have $y+y / U<p_{m+1}$. Then there are no primes in the interval $(y, y+y / U)$ so

$$
\psi(y+y / U)-\psi(y)=\sum_{y<n<y+y / U} \Lambda(n) \ll \log ^{2} x .
$$

Further for any $\eta<\varepsilon_{1}$

$$
E(y)-E(y+y / U) \ll x^{1-\alpha} \log x \ll x^{1-\eta} / U .
$$

Observing that

$$
|\exp (z)-1|<e|z| \text { for re } z<1
$$

we have

$$
\sum_{(8)} \frac{\left(e^{\delta \rho}-1\right) y^{\rho}}{\rho} \ll \delta \sum_{(8)} x^{\beta} \ll U^{-1} \int_{\sigma_{0}}^{1} x^{\sigma} N\left(\sigma, x^{\alpha}\right) \log x d \sigma .
$$

For $\sigma \in\left[\sigma_{0}, 99 / 100\right]$ we use Lemmas 3,4 and 7 , as necessary, to estimate $N\left(\sigma, x^{\alpha}\right)$. We have $\alpha G(\sigma)<1-\varepsilon_{2}$ throughout the interval and choosing $\eta=$ $\varepsilon_{2} / 300$,

$$
\int_{\sigma_{0}}^{99 / 100} x^{\sigma} N\left(\sigma, x^{\alpha}\right) \log x d \sigma \ll x^{1-e_{2} / 100+\eta} \log x \ll x / \log x
$$

LEMMA 9. For some positive constant $d, \zeta(s) \neq 0$ in the region

$$
\sigma=\operatorname{res}>1-d /(\log \tau)^{2 / 3}(\log \log \tau)^{1 / 3} \quad \text { where } \tau=|\operatorname{im} s|+2 .
$$

This is Corollary 11.4 in Montgomery [15].

We take, with some suitable constant $D$,

$$
\sigma_{1}=1-D(\log x)^{-3 / 4} \text {. }
$$

Then $\zeta(s) \neq 0$ in the region $\sigma>\sigma_{1},|t|<x^{\alpha}$ and so

$$
\int_{99 / 100}^{1} x^{\sigma} N\left(\sigma, x^{\alpha}\right) \log x d \sigma=\int_{99 / 100}^{\sigma_{1}} x^{\sigma} N\left(\sigma, x^{\alpha}\right) \log x d \sigma .
$$

For $\sigma \in\left[99 / 100, \sigma_{1}\right]$ we use Lemma 7 to estimate $N\left(\sigma, x^{\alpha}\right)$ and note that $\alpha G(\sigma)<$ $1-\varepsilon_{2}$ throughout the interval. Hence using (28),

$$
\int_{99 / 100}^{\sigma_{1}} x^{\sigma} N\left(\sigma, x^{\alpha}\right) \log x d \sigma \ll x \log ^{A+1} x \int_{99 / 100}^{\sigma_{1}} x^{-e_{2}(1-\sigma)} d \sigma \ll x / \log x .
$$

Combining (26), (27) and (29) we obtain

$$
\sum_{(8)} \frac{\left(e^{\delta \rho}-1\right) y^{\rho}}{\rho} \ll x /(U \log x) .
$$

Substituting this, (24) and (25) in (7) we obtain that for those $y$ in the range (23),

$$
|\Delta(y)|=\left|-y / U+O\left(x^{1-\eta} / U\right)+O(x / U \log x)+O\left(\log ^{2} x\right)\right|>x / 2 U \text {. }
$$


Hence

$$
\int_{x}^{2 x}|\Delta(y)|^{2} d y \gg \frac{x^{2}}{U^{2}} \sum_{\substack{x<p_{m}<2 x \\ d_{m}>x^{\mu}}} d_{m},
$$

and so, from (22),

$$
\sum d_{m} \ll x^{-2} U^{2} \int_{x}^{2 x}|\Delta(y)|^{2} d y \ll x^{F(\mu)+e}
$$

\section{REFERENCES}

1. K. Chandrasekharan, Arithmetical functions, Die Grundlehren der Math. Wissenschaften, Band 167, Springer-Verlag, New York and Berlin, 1970.

2. R. J. Cook, On the occurrence of large gaps between prime numbers, Glasgow Math. J. 20 (1979), 43-48.

3. H. Cramér, Some theorems concerning prime numbers, Ark. Mat. 15 (1920), 1-32.

4. $\ldots$ On the order of magnitude of the difference between consecutive prime numbers, Acta Arith. 2 (1937), 23-46.

5. H. Davenport, Multiplicative number theory, Markham, Chicago, Ill., 1967.

6. G. Halász and P. Turán, On the distribution of the roots of the Riemann zeta and allied functions. I, J. Number Theory 1 (1969), 121-137.

7. D. R. Heath-Brown, The differences between consecutive primes, J. London Math. Soc. 18 (1978), 7-13.

8. __ The differences between consecutive primes. II, J. London Math. Soc. 19 (1979), 207-220.

9. Z Zero-density estimates for the Riemann zeta-function and Dirichlet L-functions, J. London Math. Soc. 19 (1979), 221-232.

10. M. N. Huxley, On the differences between consecutive primes, Invent. Math. 15 (1972), 164-170.

11. A note on large gaps between prime numbers, Acta Arith. 38 (1980), 63-68.

12. A. E. Ingham, On the estimation of $N(\sigma, T)$, Quart. J. Math. Oxford Ser. 11 (1940), 291-292.

13. A. Ivić, On sums of large differences between consecutive primes, Math. Ann. 241 (1979), 1-9.

14. M. Jutila, Zero-density estimates for L-functions, Acta Arith. 32 (1977), 55-62.

15. H. L. Montgomery, Topics in multiplicative number theory, Lecture Notes in Math., vol. 227, Springer-Verlag, Berlin, Heidelberg and New York, 1971.

16. C. J. Moreno, The average size of gaps between primes, Mathematika 21 (1974), 96-100.

17. A. Selberg, On the normal density of primes in small intervals, and the difference between consecutive primes, Arch. Math. Naturvid. 47 (1943), 87-105.

18. E. C. Titchmarsh, The theory of the Riemann zeta-function, Oxford Univ. Press, Oxford, 1951.

19. R. Warlimont, Uber die Haufigkeit grosser Differenzen konsecutiver Primzahlen, Monatsh. Math. 83 (1977), 59-63.

20. D. Wolke, Grosse Differenzen zwischen aufeinanderfolgenden Primzahlen, Math. Ann. 218 (1975), 269-271.

Department of Pure Mathematics, University of Sheffield, Sheffield S10 2TN, England 